\title{
Complexity as a Novel Probe of Quantum Quenches: Universal Scalings and Purifications
}

\author{
Hugo A. Camargo, ${ }^{1,2, *}$ Pawel Caputa, ${ }^{3, \dagger}$ Diptarka Das, ${ }^{1, \ddagger}$ Michal P. Heller, ${ }^{1, \S}$ and Ro Jefferson ${ }^{1, \|}$ \\ ${ }^{1}$ Max Planck Institute for Gravitational Physics (Albert Einstein Institute), \\ Am Mühlenberg 1, 14476 Potsdam-Golm, Germany \\ ${ }^{2}$ Department of Physics, Freie Universität Berlin, Arnimallee 14, 14195 Berlin, Germany \\ ${ }^{3}$ Center for Gravitational Physics, Yukawa Institute for Theoretical Physics (YITP), \\ Kyoto University, Kitashirakawa Oiwakecho, Sakyo-ku, Kyoto 606-8502, Japan
}

(Received 18 October 2018; revised manuscript received 24 January 2019; published 27 February 2019)

\begin{abstract}
We apply the recently developed notion of complexity for field theory to a quantum quench through a critical point in $1+1$ dimensions. We begin with a toy model consisting of a quantum harmonic oscillator, and show that complexity exhibits universal scalings in both the slow and fast quench regimes. We then generalize our results to a one-dimensional harmonic chain, and show that preservation of these scaling behaviors in free field theory depends on the choice of norm. Applying our setup to the case of two oscillators, we quantify the complexity of purification associated with a subregion, and demonstrate that complexity is capable of probing features to which the entanglement entropy is insensitive. We find that the complexity of subregions is subadditive, and comment on potential implications for holography.
\end{abstract}

DOI: 10.1103/PhysRevLett.122.081601

Introduction.-Among the most exciting developments in theoretical physics is the confluence of ideas from quantum many-body systems, quantum information theory, and gravitational physics. Recent progress in this vein includes the development of tensor network methods for simulating quantum many-body systems (see, e.g., Ref. [1]), proofs of irreversibility of RG flows using quantum information techniques [2-7], and the illumination of the role of codimension-2 extremal surfaces in the emergence of holographic spacetime (see, e.g., Ref. [8]). The central technical tool in these ground-breaking results is the reduced density matrix for a spatial subregion, and the associated von Neumann entropy, cf. Refs. $[9,10]$.

However, insights from black hole physics [11-14] suggest that certain codimension-0 and codimension-1 surfaces may also play an important role in reconstructing bulk spacetime in holography, since these capture information beyond that which is accessible to the aforementioned codimension-2 surfaces - that is, beyond entanglement entropy. These geometric objects are conjectured to be dual to the "complexity" of the boundary field theory, according to the competing "complexity = volume" $(\mathrm{CV})[11,12]$ and "complexity $=$ action" $(\mathrm{CA})$ proposals $[13,14]$.

Drawing on earlier developments [15-19], Refs. [20,21] sought to make the above conjectures more precise by

Published by the American Physical Society under the terms of the Creative Commons Attribution 4.0 International license. Further distribution of this work must maintain attribution to the author(s) and the published article's title, journal citation, and DOI. Funded by SCOAP. defining the notion of complexity in (free, bosonic) quantum field theory (this idea was subsequently extended to fermionic theories in Ref. [22], see also Refs. [23,24]; for alternative approaches to defining complexity in field theories, see Refs. [25-31]). In light of the successes born of entanglement entropy mentioned above, understanding complexity in quantum field theory represents a very promising research direction. Particularly interesting open questions include the time dependence of complexity, and the interplay between complexity and entanglement entropy in nonequilibrium systems. It is therefore of value to have a tractable system in which these ideas can be concretely explored.

To that end, one of the most active areas of research into nonequilibrium quantum dynamics is the study of quantum quenches [32,33], in which remarkable progress has been made in understanding the mechanisms underlying thermalization encoded in the reduced density matrix [34]. Theoretical studies within the scope of experimental verification have revealed that smooth quenches through a critical point exhibit universal signatures via scalings. The Kibble-Zurek (KZ) scaling [35,36] is the most well-known example of this behavior, and has received a great deal of attention in recent years [37-41]. In this case, the state is evolved adiabatically until very close to the critical point, and hence the regime of KZ can be characterized as "slow." Recent studies in holography $[42,43]$, free field theory [44-46], and lattice spin models [47] have also revealed new scaling behaviors in a "fast" (nonadiabatic) regime. This fast scaling behavior appears to be a universal feature of any interacting theory which flows from a CFT in the ultraviolet (UV) [48-50]. At a technical level, previous 
studies have mainly focused on the scalings of a restricted set of one- and two-point functions, and recently on entanglement [51]. However, as we shall argue below, the latter probes at most only a spatial subsystem, while complexity is a property of the entire wave function. Hence complexity represents a means of probing features of quench dynamics to which entanglement entropy is insensitive. Initial steps towards applying complexity to quenches were taken in Ref. [52], for a quench which monotonically interpolates between two massive theories.

Motivated by these scaling phenomena, we explore the complexity of exact critical quench solutions for free scalar theories, and find evidence for universal scaling behavior. Our primary model will consist of a bosonic oscillator whose frequency varies smoothly with time, and asymptotes to a finite constant in both the far future and past. We first define complexity for a single mode, and then generalize our results to a one-dimensional harmonic chain. However, we find that a judicious definition of complexity is required in order to make the scaling expectations for free field theory manifest. Utilizing this setup, we contrast the complexity and the entanglement entropy for a fixed bipartition of the Hilbert space of two coupled harmonic oscillators. This model enables us to quantify the notion of "complexity of purification" recently introduced in Ref. [53], which allows one to associate a complexity to subregions (i.e., mixed states). We find that the complexity of subregions is subadditive, which may have interesting implications for the CV vs CA proposals above.

Complexity of quantum quenches.-Quench model: We shall begin with the following simple Hamiltonian describing a free bosonic oscillator:

$$
H(t)=\frac{1}{2 M} P^{2}+\frac{1}{2} M \tilde{\omega}^{2} X^{2},
$$

where $M$ is the mass of the oscillator, $\tilde{\omega}(t / \delta t)$ is some timedependent frequency profile with an intrinsic scale set by the parameter $\delta t$, and the canonical position and momentum operators satisfy $[X, P]=i$. However, for reasons that will become apparent below, it is preferable to work with the dimensionless variables $x \equiv \varpi X, p \equiv P / \varpi, \omega \equiv \tilde{\omega} / \varpi$, where $\varpi$ is some new mass scale, which will be given an interpretation as the gate scale when we introduce our quantum circuit (see Supplemental Material [54]). Setting $\varpi=M$ for simplicity, Eq. (1) becomes

$$
H(t)=M\left(\frac{p^{2}}{2}+\omega^{2} \frac{x^{2}}{2}\right)
$$

where the quantities appearing in the parentheses are all dimensionless, and we shall henceforth set $M=1$. The time-evolved initial ground-state wave function at time $t$ for the Hamiltonian (2) takes the form

$$
\psi_{0}(x, t)=\mathcal{N} \exp \left(\frac{i}{2} \frac{\dot{f}^{*}}{f^{*}} x^{2}\right)
$$

where $\mathcal{N} \equiv\left(2 \pi f^{*} f\right)^{-1 / 4}$, and $f(t / \delta t)$ is the solution to the equation

$$
\ddot{f}+\omega^{2} f=0 .
$$

Now, we desire a quench profile $\omega^{2}(t / \delta t)$ which admits an exact solution to this equation, and which asymptotes to a constant at both early and late times, with changes occurring in the time window $[-\delta t, \delta t]$. One of the most common profiles used in the literature (see, e.g., Ref. [51]) is

$$
\omega^{2}(t / \delta t)=\omega_{0}^{2}\left(1-\frac{1}{\cosh ^{2}\left(\frac{t}{\delta t}\right)}\right) .
$$

Here $\omega_{0}$ is a free parameter, but will gain an interpretation as the dimensionless reference-state frequency below. This profile has the property that the system is initially gapped at $t=-\infty$, but becomes gapless at $t=0$, corresponding to oscillator excitations above the ground state (3) as the system evolves via Eq. (2). In this case, the function $f(t)$ can be written explicitly in terms of hypergeometric functions; see Ref. [51].

Our interest in this setup is due to the fact that it can also be used to study the ground state of two (or more) harmonic oscillators with a time-dependent coupling. The same model was considered in Refs. [51,60,61] for investigating entanglement entropy during a quench. Explicitly, the Hamiltonian for two oscillators is given by

$$
H=\frac{1}{2}\left[p_{1}^{2}+p_{2}^{2}+2 \Omega^{2}\left(x_{1}-x_{2}\right)^{2}+\omega^{2}\left(x_{1}^{2}+x_{2}^{2}\right)\right] .
$$

In the normal-mode basis $x_{ \pm}=\left(x_{1} \pm x_{2}\right) / \sqrt{2}$, this Hamiltonian takes the decoupled form

$$
H(t)=H_{+}(t)+H_{-}(t),
$$

where the subscript denotes the use of the \pm mode in Eq. (2), with $\omega_{+}^{2}=\omega(t)^{2}$ and $\omega_{-}^{2}=\omega(t)^{2}+4 \Omega(t)^{2}$. The corresponding wave function is then given by

$$
\psi\left(x_{+}, x_{-}, t\right)=\psi_{0}\left(x_{+}, t\right) \psi_{0}\left(x_{-}, t\right),
$$

with $\psi_{0}$ given by Eq. (3). Note that this construction naturally generalizes to an $\mathrm{N}$-oscillator harmonic chain, which we will consider after introducing complexity below.

Circuit complexity: To evaluate the complexity of the target state (8), we shall apply the circuit complexity approach of Ref. [20], adapted at the level of covariance matrices as in Ref. [62]. The reader is referred to these works for details. In brief, a circuit $U$ is a unitary operator whose action on some reference state $\psi_{R}$ produces the desired target state $\psi_{T}$, 


$$
\left|\psi_{T}\right\rangle=U\left|\psi_{R}\right\rangle
$$

In analogy with quantum circuits, $U$ can be thought of as a sequence of fundamental gates, each of which effects an infinitesimal change to the state. The complexity of the target state is then defined as the length of the optimum circuit according to some suitably chosen depth function (e.g., the number of gates). Note the keyword "optimal": there may be arbitrarily many different circuits which satisfy Eq. (9). Hence the central feature of Ref. [20] was to use the geometric approach of Nielsen and collaborators [15-17] to convert the problem of finding the optimum circuit into that of identifying the minimum geodesic in the geometry generated by the algebra of gates.

Given the form of Eq. (8), it is sufficient to begin with a single oscillator. Hence we are interested in target states of the form

$$
\psi_{T}(x, t)=\left(\frac{a}{\pi}\right)^{1 / 4} \exp \left\{-\frac{1}{2}(a+i b) x^{2}\right\}
$$

where $a(t), b(t) \in \mathbb{R}$ are the real and imaginary parts of the frequency $i \dot{f}^{*} / f^{*}$ in Eq. (3), and we have suppressed the time dependence for compactness. Note that $a>0$ [one can verify that the solutions to (4) indeed satisfy this normalizability constraint], while $b$ may take any sign. Our reference state will be provided by the ground state of our time-dependent Hamiltonian (6) at $t=-\infty$,

$$
\psi_{R}(x)=\left(\frac{\omega_{R}}{\pi}\right)^{1 / 4} \exp \left(-\frac{\omega_{R}}{2} x^{2}\right),
$$

where $0<\omega_{R} \in \mathbb{R}$. Our task is now to construct a circuit $U$ satisfying (9) according to the geometric approach outlined above.

The details of our complexity calculation are given in Supplemental Material. The key point is that we may view $U$ as a matrix which acts at the level of covariance matrices, so that (9) becomes

$$
G_{\mathrm{T}}=U G_{R} U^{T},
$$

where the matrix elements of $G$ are given by

$$
G^{a b}=\left\langle\psi\left|\xi^{a} \xi^{b}+\xi^{b} \xi^{a}\right| \psi\right\rangle,
$$

where $\xi^{a} \equiv\left\{x^{1}, p^{1}, \ldots, x^{N}, p^{N}\right\}$ are the dimensionless phase-space operators for $N$ oscillators. The covariance matrix is an equivalent representation of the wave function, which has the advantage of making the explicit choice of gates more transparent. In particular, we seek the minimal set of gates necessary to effect the desired transformation. As explained in Supplemental Material [54], this naturally leads to hyperbolic space, with the metric

$$
d s^{2}=\frac{2 d z^{2}+d y^{2}}{8 z^{2}}
$$

and therefore the complexity of the target state (10) is given by the well-known geodesic distance formula on $\mathbb{t}^{2}$ (cf. Supplemental Material [54]), which admits a particularly compact expression in terms of the squeezed targetstate covariance matrix $\tilde{G}_{T}=S G_{T} S^{T}$ :

$$
\mathcal{C}=\frac{1}{2} \ln \left(\chi+\sqrt{\chi^{2}-1}\right), \quad \chi \equiv \frac{1}{2} \operatorname{tr} \tilde{G}_{T},
$$

where $S$ is the squeezing operator defined such that $S G_{R} S^{T}=\mathbb{1}$. This result immediately generalizes to the case of $N$ oscillators: since $\tilde{G}_{T}$ is block diagonal in an appropriate basis, the geometry factorizes into $N$ independent copies of $\mathbb{H}^{2}$. Hence the complexity of a onedimensional lattice of oscillators is

$$
\mathcal{C}=\sqrt{\sum_{j=1}^{N}\left[\frac{1}{2} \ln \left(\chi_{j}+\sqrt{\chi_{j}^{2}-1}\right)\right]^{2}} .
$$

Note that in this expression, we have added the complexities in the $L_{2}$ norm; we shall comment on the use of other norms in Supplemental Material [54]. By taking the continuum limit of such a lattice, we obtain the complexity for a bosonic system in $1+1$ dimensions. Specifically, we consider the harmonic chain whose Hamiltonian is given by

$$
H=\frac{1}{2} \sum_{n=1}^{N}\left[\Pi_{n}^{2}+\left(\phi_{n+1}-\phi_{n}\right)^{2}+m^{2}(t) \phi_{n}^{2}\right],
$$

where $\left(\phi_{n}, \Pi_{n}\right)$ are mutually conjugate scalar field variables. Since we work with dimensionless variables, we shall set the lattice spacing (i.e., the UV cutoff) to unity. In momentum space, each mode then satisfies

$$
\ddot{\phi_{k}}+\left(4 \sin ^{2} \frac{k}{2}+m^{2}(t)\right) \phi_{k}=0,
$$

where we have imposed periodic boundary conditions $k=k+2 \pi$, and the quench profile is given by $m(t)=$ $\omega(t / \delta t)$ in Eq. (5). The reference state, $\left|\psi_{R}\right\rangle$ is given by the ground state of the Hamiltonian (17) at $t=-\infty$ when $m(t)=\omega_{0}$. Integrating over momentum modes, the continuum limit of Eq. (16) is simply

$$
\mathcal{C}(t)=\sqrt{\int_{0}^{2 \pi} \frac{d k}{2 \pi}\left[\frac{1}{2} \ln \left(\chi_{k}(t)+\sqrt{\left.\chi_{k}^{2}(t)-1\right)}\right]^{2}\right.},
$$

where $\chi_{k}(t)$ is given in Eq. (15) with the covariance matrix corresponding to the $k$ th oscillator. 


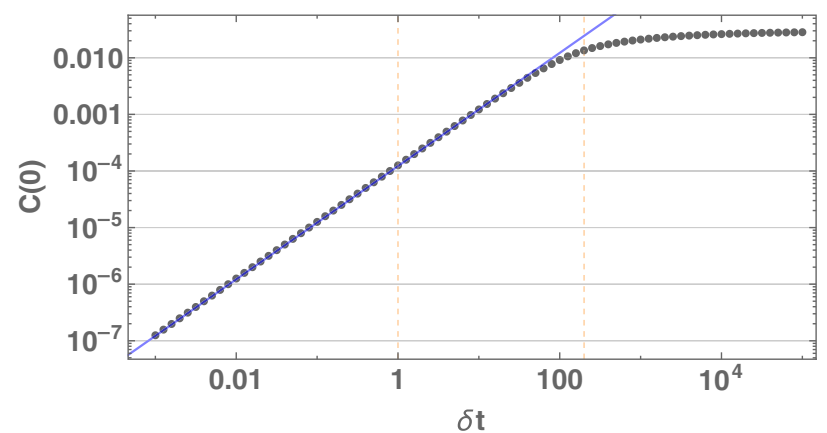

FIG. 1. Log-log plot of complexity of the $(1+1)$-dimensional free field theory (19) at the critical point $t=0 \mathrm{vs}$ the quench rate $\delta t$ (measured in units of the lattice spacing), with $\omega_{0}=0.005$. The straight-line fit (blue) reveals linear scaling in the fast regime.

Since we are interested in the behavior of complexity as the system passes through the critical point of the quench, it is sufficient to evaluate this function at $t=0$; see Fig. 1. This then allows us to extract the universal scaling behaviors, which we examine in more detail in the next section.

Universal scalings in complexity: We now wish to examine the presence of universal scalings of the critical complexity with respect to the quench rate. In particular, the contributions from individual momentum modes to $\mathcal{C}(0)$ in Eq. (19) are plotted in Fig. 2. We find that all modes go to zero in the sudden-quench limit $\delta t \rightarrow 0$, which is consistent with results for instantaneous quenches. For all $k>0$, we observe mode-dependent saturation in the slow regime $\delta t \rightarrow \infty$, consistent with what one expects from KZ. In the adiabatic approximation, the $\mathrm{KZ}$ scale arises from the Landau criterion for the breakdown of adiabaticity,

$$
\left.\frac{1}{E(t)^{2}} \frac{d E(t)}{d t}\right|_{t_{\mathrm{KZ}}}=1
$$

where $t_{\mathrm{KZ}}$ is the Kibble-Zurek time and $E$ is the timedependent mass gap from criticality. For the profile (5), one

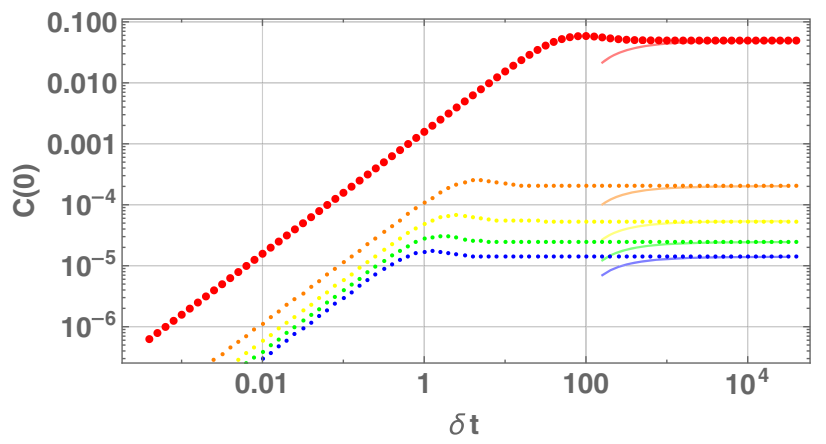

FIG. 2. Single-mode contributions to the complexity (19) at the critical point $t=0$ for $\omega_{0}=0.005$ and $k=\{0.006,0.111$, $0.216,0.320,0.425\}$ (red, orange, yellow, green, blue). For large $\delta t$, the exact solutions (dotted) agree with the saturation values (21) predicted from KZ (solid). finds $t_{\mathrm{KZ}} \approx \sqrt{\delta t / \omega_{0}}$, at which time the frequency is $\omega_{\mathrm{KZ}}(k)=$ $\sqrt{4 \sin ^{2}(k / 2)+m^{2}\left(t_{\mathrm{KZ}}\right)} \approx \sqrt{4 \sin ^{2}(k / 2)+\left(\omega_{0} / \delta t\right)}$, where we have used the fact that $m^{2}\left(t_{\mathrm{KZ}}\right) \sim \omega_{0}^{2} t_{\mathrm{KZ}}^{2} / \delta t^{2}$, since in the slow regime $\delta t>t_{\mathrm{KZ}}$ by definition. Hence the $\mathrm{KZ}$ scaling for the $k^{\text {th }}$ mode may be extracted by calculating the complexity at this frequency. One finds logarithmic KZ scaling in the slow regime for $\delta t<\left(\omega_{0} / 4\right) \csc ^{2}(k / 2)$. As soon as $\delta t$ exceeds this value, we observe saturation in the frequency (to $2 \sin (k / 2)$ ), and hence also in complexity to

$$
\mathcal{C}_{\mathrm{sat}}^{k}=\frac{1}{2} \log \left(\frac{\sqrt{\omega_{0}^{2}+2-2 \cos k}}{2\left|\sin \frac{k}{2}\right|}\right) \text {. }
$$

The $\mathrm{KZ}$ approximation is superimposed on the exact results in Fig. 2, which clearly shows agreement with the saturation value (21) in the large- $\delta t$ limit.

The critical complexity of the zero mode $k=0$ exhibits universal scalings in both the slow and fast regimes. Indeed, this same behavior is exhibited by the single quantum oscillator we initially introduced upon sending the frequency to zero (i.e., we take the $\omega_{-}$solution for the twooscillator case above). Unlike higher modes, the zero mode does not saturate at large $\delta t$ since the logarithmic scaling is always present. From the KZ analysis above, we can derive the universal coefficient of the $\log$ as $\frac{1}{4}$, which is confirmed by fitting the exact solution, as shown in Fig. 3. We note that the $\mathrm{KZ}$ scaling exhibited by entanglement entropy under a critical quantum quench has the same form, but with a $\frac{1}{6}$ coefficient instead [51,63]. Meanwhile in the fast regime ( $\delta t<1$ in lattice units), the complexity grows linearly with $\delta t$. While these scalings are present for higher modes as well, they are confined to increasingly narrow regions of $\delta t$ for larger values of $k$.

Complexity vs entanglement. - One of the main motivations for the holographic complexity proposals was the observation that the information contained in the reduced

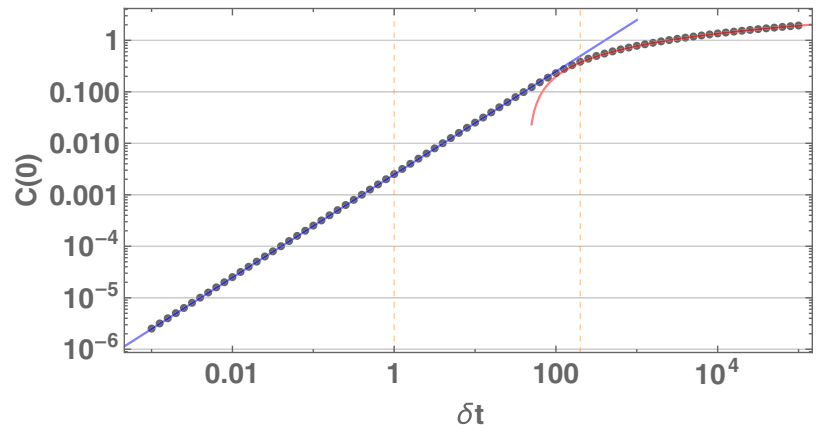

FIG. 3. Zero-mode contribution to $\mathcal{C}(0)$ (19) as a function of the quench rate $\omega_{0} \delta t$, with $\omega_{0}=0.005$. The complexity scales linearly in the fast regime $(\ln C / \ln \delta t=1$, blue), and smoothly transitions to a logarithmic scaling $\frac{1}{4} \log \delta t$ in the slow regime (red). The transition to $\mathrm{KZ}$ occurs at $\omega_{0} \delta t \sim 1$, which in this case is $\delta t \sim 200$ in lattice units. 
density matrix of any spatial bipartition of the CFT Hilbert space, as encoded in the entanglement entropy, is generally insufficient to determine the entire bulk geometry [12] (see also Ref. [64] and references therein). One can then ask whether complexity provides another take on the information contained in reduced density matrices. Indeed, recent proposals for the complexity of subregions in holography - that is, on the bulk side - have been made in Refs. [65-69]. However, since the field-theoretic notion of complexity above is defined for pure states, it is not a priori clear how to define complexity for the reduced density matrix corresponding to some spatial subregion.

A particularly natural extension of existing pure-state definitions to this case is the complexity of purification, recently outlined in Ref. [53], in which the complexity of the subsystem is defined by minimizing over the complexities of all possible purifications (see also Ref. [70]). Applying our quench setup above to the case of two oscillators allows us to quantify this proposal, by considering the reduced density matrix corresponding to a single oscillator, say $x_{1}$, and purifying within the original Hilbert space of Gaussian states (i.e., without ancilla). The total wave function depends on six real parameters, three of which we fix by our knowledge of the covariance matrix for oscillator $x_{1}$. Minimizing over the remaining three parameters then gives the complexity of purification for the subsystem, which we shall denote $\mathcal{C}_{A}$ in reference to a generic subsystem $A$ and its complement $\bar{A}$.

As observed in Fig. 4, the complexity of purification satisfies $\mathcal{C} / 2 \leq \mathcal{C}_{A} \leq \mathcal{C}$, which we have verified numerically for a wide range of values in the six-parameter landscape spanned by the components of the covariance matrix. The upper inequality is saturated if and only if the original target state happens to be the least complex state among all possible purifications. Meanwhile, the lower inequality is

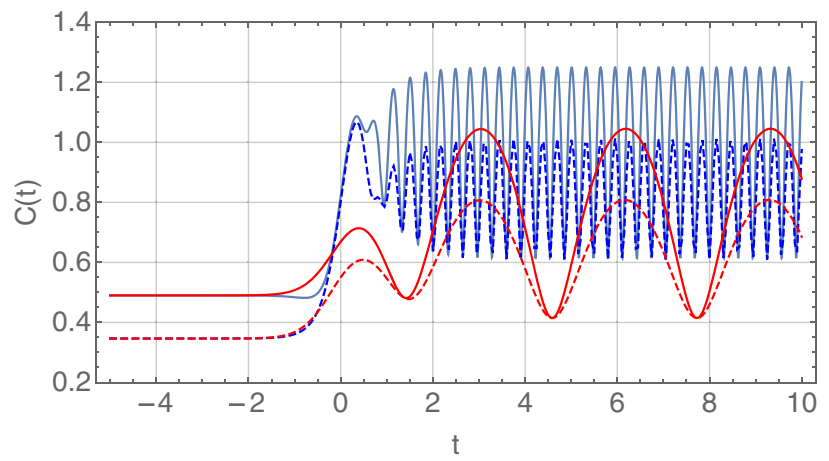

FIG. 4. Comparison of the complexity (16) as a function of time $t$ of the original target state (solid) and the optimum purification (dashed) for $\delta t=10$ (blue) and $\delta t=1$ (red), with $\omega_{R}=0.5$ for both oscillators. Note that the latter never exceeds the former, and is always greater than $\mathcal{C} / 2$; that is, the complexity of purification appears to satisfy superadditivity (22). We have tested this conjecture numerically for $\sim 70000$ cases. saturated if and only if the original target state is a product state with respect to the chosen bipartition; i.e., subsystem $A$ describes a pure state, $S_{A}=0$. This can be understood from the fact that the purification process seeks to produce a state which is as close to the reference state as possible, since the latter has minimum complexity by fiat. Since in this case the reference state is an unentangled product state, the minimum purification is one in which the complement $\bar{A}$ is also an unentangled product state-but this is only possible if the original state is a tensor product of the form $\mathcal{H}_{A} \otimes \mathcal{H}_{\bar{A}}$; otherwise the entanglement across the bipartition prevents one from obtaining the reference state in the restriction $\bar{A}$. While one should exercise caution in blithely generalizing from this simple two-oscillator case, the above leads us to suggest that the complexity of subsystems is subadditive:

$$
\mathcal{C}_{A}+\mathcal{C}_{\bar{A}} \geq \mathcal{C}
$$

As observed in Ref. [53], this agrees with the holographic CA proposal, but not with the CV proposal, which is superadditive.

Outlook.-Quenches represent tractable models of dynamical quantum systems in which complexity can be better understood, as well as yield new physical insights; e.g., we have found that complexity can be used to extract universal scalings. We have also examined the complexity of subregions (i.e., mixed states) via their purifications. Since complexity encodes global information about the state, it is sensitive to features to which entanglement is blind. We find that subregion complexity appears to satisfy superadditivity (22), which is consistent with the CA proposal. While it would be premature to take definitive lessons for holography from such simple free-field models, this may provide further hints as to the proper notion of complexity in holographic field theories, and thereby shed light on ongoing efforts to reconstruct bulk spacetime in AdS/CFT.

We thank E. M. Brehm, S. R. Das, D. A. Galante, E. López, J. Magan, T. Takayanagi, M. Walter, and the coauthors of Ref. [62] for helpful conversations. We would especially like to thank H. Marrochio, R. C. Myers, and M. Smolkin for comments on a draft of this manuscript. H. C., D. D., M. H., and R. J. acknowledge the hospitality of MITP during the "Modern Techniques for AdS and CFT" program during the completion of this project. H. C. is partially supported by the Konrad-Adenauer-Stiftung through their Sponsorship Program for Foreign Students. P. C. is supported by the Simons Foundation through the "It from Qubit" collaboration and by the JSPS starting grant KAKENHI 17H06787. The Gravity, Quantum Fields and Information group at AEI is generously supported by the Alexander von Humboldt Foundation and the Federal Ministry for Education and Research through the Sofja Kovalevskaja Award. 
*hugo.camargo@aei.mpg.de

pawel.caputa@yukawa-kyoto-u.ac.jp

*diptarka.das@aei.mpg.de

§michal.p.heller@aei.mpg.de

On leave from National Centre for Nuclear Research, Hoża 69, 00-681 Warsaw, Poland.

"rjefferson@aei.mpg.de

[1] R. Orus, Ann. Phys. (Amsterdam) 349, 117 (2014).

[2] H. Casini and M. Huerta, Phys. Rev. D 85, 125016 (2012).

[3] H. Casini and M. Huerta, Phys. Lett. B 600, 142 (2004).

[4] R. C. Myers and A. Sinha, Phys. Rev. D 82, 046006 (2010).

[5] R. C. Myers and A. Sinha, J. High Energy Phys. 01 (2011) 125.

[6] H. Casini, M. Huerta, R. C. Myers, and A. Yale, J. High Energy Phys. 10 (2015) 003.

[7] H. Casini, E. Teste, and G. Torroba, Phys. Rev. Lett. 118, 261602 (2017).

[8] M. Rangamani and T. Takayanagi, Lect. Notes Phys. 931, 1 (2017).

[9] S. Ryu and T. Takayanagi, Phys. Rev. Lett. 96, 181602 (2006).

[10] V. E. Hubeny, M. Rangamani, and T. Takayanagi, J. High Energy Phys. 07 (2007) 062.

[11] L. Susskind, Fortschr. Phys. 64, 44 (2016); 64, 24 (2016).

[12] L. Susskind, Fortschr. Phys. 64, 49 (2016).

[13] A. R. Brown, D. A. Roberts, L. Susskind, B. Swingle, and Y. Zhao, Phys. Rev. Lett. 116, 191301 (2016).

[14] A. R. Brown, D. A. Roberts, L. Susskind, B. Swingle, and Y. Zhao, Phys. Rev. D 93, 086006 (2016).

[15] M. A. Nielsen, arXiv:quant-ph/0502070.

[16] M. A. Nielsen, M. R. Dowling, M. Gu, and A. M. Doherty, Science 311, 1133 (2006).

[17] M. A. Nielsen and M. R. Dowling, arXiv:quant-ph/0701004.

[18] J. Haegeman, T. J. Osborne, H. Verschelde, and F. Verstraete, Phys. Rev. Lett. 110, 100402 (2013).

[19] M. Nozaki, S. Ryu, and T. Takayanagi, J. High Energy Phys. 10 (2012) 193.

[20] R. Jefferson and R. C. Myers, J. High Energy Phys. 10 (2017) 107.

[21] S. Chapman, M. P. Heller, H. Marrochio, and F. Pastawski, Phys. Rev. Lett. 120, 121602 (2018).

[22] L. Hackl and R. C. Myers, J. High Energy Phys. 07 (2018) 139.

[23] R. Khan, C. Krishnan, and S. Sharma, Phys. Rev. D 98, 126001 (2018).

[24] A. P. Reynolds and S. F. Ross, Classical Quantum Gravity 35, 095006 (2018).

[25] P. Caputa, N. Kundu, M. Miyaji, T. Takayanagi, and K. Watanabe, Phys. Rev. Lett. 119, 071602 (2017).

[26] P. Caputa, N. Kundu, M. Miyaji, T. Takayanagi, and K. Watanabe, J. High Energy Phys. 11 (2017) 097.

[27] B. Czech, Phys. Rev. Lett. 120, 031601 (2018).

[28] A. Bhattacharyya, P. Caputa, S. R. Das, N. Kundu, M. Miyaji, and T. Takayanagi, J. High Energy Phys. 07 (2018) 086.

[29] J. M. Magan, J. High Energy Phys. 09 (2018) 043.

[30] P. Caputa and J. M. Magan, arXiv:1807.04422.

[31] R.-Q. Yang, Y.-S. An, C. Niu, C.-Y. Zhang, and K.-Y. Kim, Eur. Phys. J. C 79, 109 (2019).
[32] M. Gring, M. Kuhnert, T. Langen, T. Kitagawa, B. Rauer, M. Schreitl, I. Mazets, D. A. Smith, E. Demler, and J. Schmiedmayer, Science 337, 1318 (2012).

[33] A. Polkovnikov, K. Sengupta, A. Silva, and M. Vengalattore, Rev. Mod. Phys. 83, 863 (2011).

[34] M. Rigol, V. Dunjko, and M. Olshanii, Nature (London) 452, 854 (2008).

[35] T. W. B. Kibble, J. Phys. A 9, 1387 (1976).

[36] W. H. Zurek, Nature (London) 317, 505 (1985).

[37] A. Chandran, A. Erez, S. S. Gubser, and S. L. Sondhi, Phys. Rev. B 86, 064304 (2012).

[38] S. Mondal, D. Sen, and K. Sengupta, Quantum Quenching, Annealing and Computation, Lecture Notes in Physics, Vol. 802 (Springer, Berlin, Heidelberg, 2010).

[39] V. Gritsev and A. Polkovnikov, in Understanding Quantum Phase Transitions, edited by Lincoln D. Carr (Taylor \& Francis, Boca Raton, 2010).

[40] J. Dziarmaga, Adv. Phys. 59, 1063 (2010).

[41] A. Lamacraft and J. Moore, Ultracold Bosonic and Fermionic Gases, Contemporary Concepts in Condensed Matter Science (Elsevier, New York, 2012).

[42] A. Buchel, L. Lehner, and R. C. Myers, J. High Energy Phys. 08 (2012) 049.

[43] A. Buchel, L. Lehner, R. C. Myers, and A. van Niekerk, J. High Energy Phys. 05 (2013) 067.

[44] A. Buchel, R. C. Myers, and A. van Niekerk, Phys. Rev. Lett. 111, 201602 (2013).

[45] S. R. Das, D. A. Galante, and R. C. Myers, Phys. Rev. Lett. 112, 171601 (2014).

[46] S. R. Das, D. A. Galante, and R. C. Myers, J. High Energy Phys. 02 (2015) 167.

[47] D. Das, S. R. Das, D. A. Galante, R. C. Myers, and K. Sengupta, J. High Energy Phys. 11 (2017) 157.

[48] S. R. Das, D. A. Galante, and R. C. Myers, J. High Energy Phys. 05 (2016) 164.

[49] M. Goykhman, T. Shachar, and M. Smolkin, J. High Energy Phys. 06 (2018) 168.

[50] A. Dymarsky and M. Smolkin, J. High Energy Phys. 01 (2018) 112.

[51] P. Caputa, S. R. Das, M. Nozaki, and A. Tomiya, Phys. Lett. B 772, 53 (2017).

[52] D. W. F. Alves and G. Camilo, J. High Energy Phys. 06 (2018) 029.

[53] C. A. Agón, M. Headrick, and B. Swingle, arXiv:1804. 01561.

[54] See Supplemental Material at http://link.aps.org/ supplemental/10.1103/PhysRevLett.122.081601 for more details, which is split into three sections and contains Refs. [55-59]. Section A contains the details of the circuit complexity calculation, and derives the associated geometry. Section B explains the geometrical embedding into anti-de Sitter space. Section C explains the calculation of the complexity of purification.

[55] H. A. Camargo, L. Hackl, M. P. Heller, and B. Swingle.

[56] M. Moosa, J. High Energy Phys. 03 (2018) 031.

[57] S. Chapman, H. Marrochio, and R. C. Myers, J. High Energy Phys. 06 (2018) 046.

[58] S. Chapman, H. Marrochio, and R. C. Myers, J. High Energy Phys. 06 (2018) 114. 
[59] D. S. Ageev, I. Ya. Aref'eva, A. A. Bagrov, and M. I. Katsnelson, J. High Energy Phys. 08 (2018) 071.

[60] L. Bombelli, R. K. Koul, J. Lee, and R. D. Sorkin, Phys. Rev. D 34, 373 (1986).

[61] M. Srednicki, Phys. Rev. Lett. 71, 666 (1993).

[62] S. Chapman, J. Eisert, L. Hackl, M. P. Heller, R. Jefferson, H. Marrochio, and R. C. Myers, arXiv:1810.05151.

[63] M. Nishida, M. Nozaki, Y. Sugimoto, and A. Tomiya, arXiv: 1712.09899.

[64] B. Freivogel, R. A. Jefferson, L. Kabir, B. Mosk, and I.-S. Yang, Phys. Rev. D 91, 086013 (2015).
[65] D. Carmi, R. C. Myers, and P. Rath, J. High Energy Phys. 03 (2017) 118.

[66] M. Alishahiha, Phys. Rev. D 92, 126009 (2015).

[67] O. Ben-Ami and D. Carmi, J. High Energy Phys. 11 (2016) 129.

[68] R. Abt, J. Erdmenger, H. Hinrichsen, C. M. MelbyThompson, R. Meyer, C. Northe, and I. A. Reyes, Fortschr. Phys. 66, 1800034 (2018).

[69] R. Abt, J. Erdmenger, M. Gerbershagen, C. M. MelbyThompson, and C. Northe, J. High Energy Phys. 01 (2019) 012.

[70] H. Stoltenberg, Phys. Rev. D 98, 126012 (2018). 